\title{
Front Matter: Volume 10953
}

, "Front Matter: Volume 10953," Proc. SPIE 10953, Medical Imaging 2019: Biomedical Applications in Molecular, Structural, and Functional Imaging, 1095301 (11 June 2019); doi: 10.1117/12.2533275

SPIE. Event: SPIE Medical Imaging, 2019, San Diego, California, United States 


\title{
Medical Imaging 2019
}

\section{Biomedical Applications in Molecular, Structural, and Functional Imaging}

\author{
Barjor Gimi \\ Andrzej Krol \\ Editors
}

19-21 February 2019

San Diego, California, United States

Sponsored by

SPIE

Cooperating Organizations

AAPM-American Association of Physicists in Medicine (United States)

IFCARS—International Foundation for Computer Assisted Radiology and Surgery (Germany)

MIPS-Medical Image Perception Society (United States)

SIIM-Society for Imaging Informatics in Medicine (United States)

WMIS-World Molecular Imaging Society

Published by

SPIE 
The papers in this volume were part of the technical conference cited on the cover and title page. Papers were selected and subject to review by the editors and conference program committee. Some conference presentations may not be available for publication. Additional papers and presentation recordings may be available online in the SPIE Digital Library at SPIEDigitalLibrary.org.

The papers reflect the work and thoughts of the authors and are published herein as submitted. The publisher is not responsible for the validity of the information or for any outcomes resulting from reliance thereon.

Please use the following format to cite material from these proceedings:

Author(s), "Title of Paper," in Medical Imaging 2019: Biomedical Applications in Molecular, Structural, and Functional Imaging, edited by Barjor Gimi, Andrzej Krol, Proceedings of SPIE Vol. 10953 (SPIE, Bellingham, WA, 2019) Seven-digit Article CID Number.

ISSN: 1605-7422

ISSN: $2410-9045$ (electronic)

ISBN: 9781510625532

ISBN: 9781510625549 (electronic)

Published by

SPIE

P.O. Box 10, Bellingham, Washington 98227-0010 USA

Telephone +1360 676 3290 (Pacific Time) · Fax +1 3606471445

SPIE.org

Copyright @ 2019, Society of Photo-Optical Instrumentation Engineers.

Copying of material in this book for internal or personal use, or for the internal or personal use of specific clients, beyond the fair use provisions granted by the U.S. Copyright Law is authorized by SPIE subject to payment of copying fees. The Transactional Reporting Service base fee for this volume is $\$ 18.00$ per article (or portion thereof), which should be paid directly to the Copyright Clearance Center (CCC), 222 Rosewood Drive, Danvers, MA 01923. Payment may also be made electronically through CCC Online at copyright.com. Other copying for republication, resale, advertising or promotion, or any form of systematic or multiple reproduction of any material in this book is prohibited except with permission in writing from the publisher. The CCC fee code is 1605$7422 / 19 / \$ 18.00$.

Printed in the United States of America by Curran Associates, Inc., under license from SPIE.

Publication of record for individual papers is online in the SPIE Digital Library.

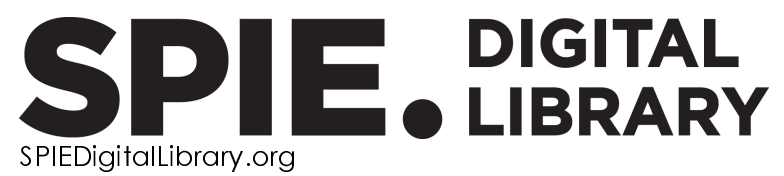

Paper Numbering: Proceedings of SPIE follow an e-First publication model. A unique citation identifier (CID) number is assigned to each article at the time of publication. Utilization of CIDs allows articles to be fully citable as soon as they are published online, and connects the same identifier to all online and print versions of the publication. SPIE uses a seven-digit CID article numbering system structured as follows:

- The first five digits correspond to the SPIE volume number.

- The last two digits indicate publication order within the volume using a Base 36 numbering system employing both numerals and letters. These two-number sets start with $00,01,02,03,04$, 05, 06, 07, 08, 09, 0A, OB ... 0Z, followed by 10-1Z, 20-2Z, etc. The CID Number appears on each page of the manuscript. 


\title{
Contents
}

\author{
ix Authors \\ xiii Conference Committee \\ xv 2019 Medical Imaging Award Recipients \\ xvii Introduction
}

SESSION 1 NOVEL IMAGING TECHNIQUES AND APPLICATIONS I

1095302 Rapid cone-beam computed tomography using an ultra-high frame rate imaging photon counting detector (PCD) with $100 \mu \mathrm{m}$ resolution [10953-1]

$1095303 \quad$ Towards 50 ps TOF-PET for brain imaging [10953-2]

1095304 Design, fabrication and evaluation of non-imaging, label-free pre-screening tool using quantified bio-electrical tissue profile [10953-3]

1095306 Investigation of Pockels effect in optical property modulation-based radiation detection method for positron emission tomography [10953-5]

\section{SESSION 2 KEYNOTE AND OPTICAL/VASCULAR I}

1095308 Deep learning based approach for fully automated detection and segmentation of hard exudate from retinal images [10953-7]

1095309 Deep convolutional network based on rank learning for OCT retinal images quality assessment [10953-8]

10953 OA Rapid sequence angiography with a 3D printed aneurysm phantom and an ultra-high frame rate imaging photon counting detector (PCD) [10953-83]

\section{SESSION $3 \quad$ NEUROLOGICAL IMAGING I}

10953 OB Investigating a quantitative radiomics approach for brain tumor classification [10953-9]

10953 OC Progressive degeneration of white matter functional connectivity in Alzheimer's disease [10953-10]

10953 OD Phase fMRI reveals sparser function connectivity than magnitude fMRI [10953-1 1] 
10953 OE Estimation of axonal conduction speed and the inter hemispheric transfer time using connectivity informed maximum entropy on the mean [10953-12]

10953 OF Quantitative assessment of dMRI-based dentate-rubro-thalamic tractography in squirrel monkey [10953-13]

\section{SESSION 4 PULMONARY}

10953 0G Pulmonary blood vessels extraction from dual-energy CT images using a synchrotron radiation micro-CT [10953-14]

$10953 \mathrm{OH} \quad$ Texture analysis of thoracic CT to predict hyperpolarized gas MRI lung function [10953-15]

$10953 \mathrm{Ol} \quad$ Micro-computed tomography imaging of cigarette smoke-exposed mice to model early chronic obstructive pulmonary disease (COPD) [10953-16]

$10953 \mathrm{OJ} \quad$ Development and evaluation of pulmonary imaging multi-parametric response maps for deep phenotyping of chronic obstructive pulmonary disease [10953-17]

\section{SESSION 5 INNOVATIONS IN IMAGE PROCESSING I}

10953 OK Multiseg pipeline: automatic tissue segmentation of brain MR images with subject-specific atlases [10953-18]

$10953 \mathrm{OL} \quad$ Unsupervised segmentation of micro-CT images based on a hybrid of variational inference and adversarial learning [10953-19]

10953 OM Developing a computer-aided image analysis and visualization tool to predict region-specific brain tissue "at risk" for developing acute ischemic stroke [10953-20]

10953 ON Large-scale parcellation of the ventricular system using convolutional neural networks [10953-21]

$1095300 \quad$ Effective 3D humerus and scapula extraction using low-contrast and high-shape-variability MR data [10953-22]

\section{SESSION $6 \quad$ INNOVATIONS IN IMAGE PROCESSING II}

10953 OP Coupled active shape models for automated segmentation and landmark localization in high-resolution CT of the foot and ankle [10953-23]

$109530 Q \quad$ Skin lesion boundary segmentation with fully automated deep extreme cut methods [10953-24]

10953 OR The effect of color constancy algorithms on semantic segmentation of skin lesions [10953-25] 
10953 OS Using deep learning to detect oesophageal lesions in PET-CT [10953-26]

10953 От A web-based system for statistical shape analysis in temporomandibular joint osteoarthritis [10953-27]

10953 OU Measuring hippocampal neuroanatomical asymmetry to better diagnose Alzheimer's disease [10953-28]

10953 OV Improving estimates of brain metabolite concentrations in MR spectroscopic imaging (MRSI) through MRI content [10953-29]

10953 OW Electrical impedance mapping for localizing evolving traumatic brain injury [10953-30]

10953 OX Extraction of co-expressed discriminative features of schizophrenia in imaging epigenetics framework [10953-31]

10953 OY Substantia nigra segmentation on neuromelanin-sensitive MRI [10953-32]

10953 OZ Pseudo-CT image generation from mDixon MRI images using fully convolutional neural networks [10953-33]

$1095310 \quad$ Imaging inhibitory effect of fissure sealants on demineralization of adjacent enamel with cross polarization OCT [10953-34]

1095311 Spatial arrangement of leakage patterns in diabetic macular edema is associated with tolerance of aflibercept treatment interval length: preliminary findings [10953-35]

1095312 Morphology of vascular network in eyes with diabetic macular edema varies based on tolerance of aflibercept treatment interval length: preliminary findings [10953-36]

$1095313 \quad$ Imaging of murine melanoma tumors using fluorescent gold nanoparticles [10953-37]

$1095314 \quad$ Initial assessment of neuro pressure gradients in carotid stenosis using 3D printed patient-specific phantoms [10953-38]

109515 Toward an automatic segmentation of mitral valve chordae [10953-39] 
109516 Methods for quantitative characterization of bone injury from computed-tomography images [10953-40]

109517 Quantitative evaluation of bone microstructure using high-resolution extremity cone-beam CT with a CMOS detector [10953-41]

1095318 Advanced statistical analysis to classify high dimensionality textural probability-distribution matrices [10953-42]

$109531 \mathrm{~A} \quad$ Quantitative cartilage imaging using spectral photon-counting detector based computed tomography [10953-44]

\section{SESSION $10 \quad$ MRI AND FMRI}

10953 1B Auto-labeling of respiratory time points in free-breathing thoracic dynamic MR image acquisitions for 4D image construction [10953-45]

$109531 \mathrm{C}$ Semi-automated myocardial segmentation in native T1-mapping CMR using deformable nonrigid registration of CINE images [10953-46]

10953 1D Classification of autism spectrum disorder from resting-state fMRI with mutual connectivity analysis [10953-47]

$109531 \mathrm{E} \quad$ Automated signal drift and global fluctuation removal from 4D fMRI data based on principal component analysis as a major preprocessing step for fMRI data analysis [10953-48]

$109531 \mathrm{~F} \quad$ High-resolution MRI of the mouse cerebral vasculature to study hemodynamic-induced vascular remodeling [10953-49]

SESSION 11 NOVEL IMAGING TECHNIQUES AND APPLICATIONS II

$109531 \mathrm{G}$ Tomosynthesis method for depth resolution of beta emitters [10953-50]

$109531 \mathrm{H} \quad$ To gate or not to gate: an evaluation of respiratory gating techniques to improve volume measurement of murine lung tumors in micro-CT imaging [10953-51]

$1095311 \quad$ Scanning, registration, and fiber estimation of rabbit hearts using micro-focus and refraction-contrast $x$-ray $\mathbf{C T}$ [10953-52]

$109531 \mathrm{~J} \quad$ Demonstration of improved image resolution for larger focal spot sizes by decreasing anode angles in clinical settings [10953-53]

$109531 \mathrm{~K} \quad$ Novel measurement of LV twist using 4DCT: quantifying accuracy as a function of image noise [10953-54] 
$109531 \mathrm{~L} \quad$ An MR compatible aortic arch phantom with calcific polymeric valves [10953-55]

$109531 \mathrm{M} \quad$ A learning-based automatic segmentation method on left ventricle in SPECT imaging [10953-56]

$109531 \mathrm{~N} \quad$ Using FDG and NaF PET/CT imaging to investigate the relationship between inflammation and microcalcification in the aorta [10953-57]

1095310 Improved reproducibility of calcium mass score using deconvolution and partial volume correction [10953-58]

$109531 \mathrm{P} \quad$ Comparison of benchtop pressure gradient measurements in 3D printed patient specific cardiac phantoms with CT-FFR and computational fluid dynamic simulations [10953-59]

POSTERS: OPTICAL AND OCULAR IMAGING

$109531 Q \quad$ Effect of silver nitrate on interfacial gap detection under polymeric dental restoration in CP-OCT imaging [10953-60]

\section{POSTERS: IMAGE PROCESSING}

$109531 \mathrm{~T} \quad$ A comparative study of graph search algorithms for segmenting coronary arteries from cine angiography [10953-63]

$109531 \mathrm{U} \quad$ 2D pattern matching of frontal plane radiograph to 3D model identifies structural and functional deficiencies of spinal pelvic system in consideration of mechanical spine pain [10953-64]

$109531 \mathrm{~V} \quad$ Toward employing the full potential of magnetic particle imaging: exploring visualization techniques and clinical use cases for real-time 3D vascular imaging [10953-65]

10953 IW Brain MRI classification based on machine learning framework with auto-context model [10953-66]

10953 1X Learning 3D non-rigid deformation based on an unsupervised deep learning for PET/CT image registration [10953-67]

$109531 Y \quad$ A combined deep-learning approach to fully automatic left ventricle segmentation in cardiac magnetic resonance imaging [10953-68]

$109531 \mathrm{X}$ XNet: a convolutional neural network (CNN) implementation for medical x-ray image segmentation suitable for small datasets [10953-69]

1095320 Automatic pressure ulcer measurement using RGB-D data [10953-70]

$1095321 \quad$ Automatic delineation of anterior and posterior cruciate ligaments by combining deep learning and deformable atlas based segmentation [10953-71] 
1095322 Computerized assessment of glaucoma severity based on color fundus images [10953-72]

1095325 Cascaded convolutional neural networks for spine chordoma tumor segmentation from MRI [10953-75]

POSTERS: NEUROLOGICAL IMAGING

1095326 Conformal initialization for shape analysis applications in SlicerSALT [10953-77]

1095329 Machine-learning based classification of glioblastoma using dynamic susceptibility enhanced MR image [10953-80]

10953 2A Predicting conversion to psychosis in clinical high risk patients using resting-state functional MRI features [10953-84]

POSTERS: NOVEL IMAGING TECHNIQUES AND APPLICATIONS

10953 2B Investigation of necessary conditions for imaging cell analysis using EIT [10953-81]

10953 2C Study of separation between ex vivo malignant and benign prostatic tissue using magnetic resonance electrical property tomography [10953-82] 


\section{Authors}

Numbers in the index correspond to the last two digits of the seven-digit citation identifier (CID) article numbering system used in Proceedings of SPIE. The first five digits reflect the volume number. Base 36 numbering is employed for the last two digits and indicates the order of articles within the volume. Numbers start with 00, 01, 02, 03, 04, 05, 06, 07, 08, 09, OA, OB...0Z, followed by 10-12, 20-2Z, etc.

Abidin, Anas Z., OB, 1D

Ackerley, I., OS

Aghaei, Faranak, 04, OM

Akita, Toshiaki, 11

Alavi, Abass, $1 \mathrm{~N}$

Ali, Arif, 29

Amini, Amir A., 1L, $1 \mathrm{~T}$

Anderson, Adam W., OC, OF

Ando, Masami, 11

Angel, Erin, 1P

Antani, Sameer K., 1E

Appenzeller, Simone, OV

Arridge, Simon, $1 \mathrm{G}$

Assunção, Antonildes N. Jr., $1 Y$

Atlason, Hans E., ON

Auslander, Thomas, $1 \mathrm{~N}$

Badea, C. T., 1H

Bai, Yuntong, OX

Bakhsh, Turki A., 1Q

Barker, Andrea L., OJ

Bateman, Ted, 16

Batzdorf, Alexandra, 1N

Baver, David F., OW

Bednarek, D. R., 02, 0A, $1 \mathrm{~J}$

Benavides, Erika, 18

Benike, Amy, $1 \mathrm{~A}$

Berger, Marie-Odile, 15

Bianchi, Jonas, OT

Blocker, S. J., $1 \mathrm{H}$

Bobba, Vishal, 11, 12

Bordignom, Adriano, $1 Y$

Braman, Nathaniel, 12

Brehler, M., OP, 17

Breighner, R. E., 17

Brück, Rainer, 2B

Bullock, Joseph, 1 Z

Butman, John A., 25

Calhoun, Vince D., OD, OX

Callahan, Sean, $1 \mathrm{~L}$

Calvert, Nick, $1 G$

Campbell, Robert M., 1B

Cao, Q., 17

Capaldi, Dante P. I., OH, OJ

Carrino, J. A., 17

Carvalho, Talles, $1 Y$

Cecchi, Guillermo, 2A

Celaya-Padilla, José M., OU

Cevidanes, Lucia, OT, 18

Chandra, Anusha Ramesh, $1 \mathrm{~F}$
Chen, Hang, 22

Chen, Zikuan, OD

Claassen, Daniel, OF

Colvert, Brendan, $1 \mathrm{~K}$

Colvert, Gabrielle M., 1K

Contijoch, Francisco, $1 \mathrm{~K}$

Cowan, L., 20

Cuesta-Lázaro, Carolina, 12

Curran, Walter J., 1M, 1W, 29

Danala, Gopichandh, OM

Dantas, Roberto N. Jr., IY

Dar, Irfaan, OB

Davies, Jason M., 14

Dawant, Benoit, OF

Demehri, S., OP

Deriche, Rachid, OE

de Sá Rebelo, Marina F. S., $1 Y$

Deslauriers-Gauthier, Samuel, OE

Ding, Zhaohua, OC

Dong, Xue, IW

do Val, Renata, 1 Y

D'Souza, Adora M., OB, 1D

Eck, Brendan L., 10

Eddy, Rachel L., OJ

Ehlers, Justis, 11, 12

Ellingsen, Lotta M., ON

Everitt, Alicia, OW

Fan, Zhaoyang, OY

Fang, Zhengyang, 26

Farrag, Nadia A., 1C

Feng, Jun, 09

Fenster, Aaron, $\mathrm{OH}$

Figueredo, Natalia, 11, 12

Fontolan, Juliana, OV

Ford, Nancy L., Ol, 13

Fujita, Hiroshi, $1 \mathrm{X}$

Fuketa, S., OG

Gao, Yurui, OC, OF

Ghafghazi, Shahab, $1 T$

Gibas, Christian, 2B

Goldgof, D., 20

Gonçalves, João Roberto, OT

Gore, John C., OC

Goyal, Manu, OQ, OR

Gudnason, Vilmundur, ON

Guo, Shuxu, 1B

Gutierrez, Liza C., 1F

Gutierrez, Marco A., IY

Hall, K., 20 
Halling-Brown, M., OS

Halter, Ryan J., OW, 2C

Hara, Takeshi, $1 \mathrm{X}$

Harmon, Eric S., 03

Harms, Joseph, $1 \mathrm{M}$

$\mathrm{He}$, Xiaoxiao, $0 \mathrm{O}$

Heidari, Morteza, 04, OM

Henn, Alex, $1 \mathrm{~L}$

Hernandez-Cerdan, Pablo, 16, 18

Hewitt, Brett, OR

Hogg, Elliot, OY

Høilund-Carlsen, Poul F., 1N

Holbrook, M., 1H

Hord, William, 2A

Huang, Chao, OT

Hyams, Elias S., 2C

Ichihara, Shu, 11

Ionita, Ciprian N., 02, 14, 1F, 1P

loshida, Marcos, OT

Islam, A., OP

Itoh, H., OG

lyer, Vijay, IP

J., Vijayananda, 21

Jeong, Jiwoong Jason, 29

Ji, Bing, 29

Jiang, Huiyan, $1 X$

Jiang, Xiaojun, IW

Jones, Brandon, $1 \mathrm{~N}$

Kang, Hongjian, $1 \mathrm{X}$

Kawata, Y., OG

Kendrick, Michael, $1 \mathrm{~L}$

Kheradvar, Arash, 1L

Knopp, Tobias, $1 \mathrm{~V}$

Kozomara, Steve, 13

Krebs, J., 02, 0A

Krol, Andrzej, 03

Kumamaru, Kanako Kunishima, 1P

Landman, Bennett, OF

Lee, lan, 01

Lei, Yang, 1M, 1W, 29

Leng, Shuai, $1 \mathrm{~A}$

Levi, Jacob, 10

Levy, Elad I., 14

Lewis, E., OS

Li, Debiao, OY

Li, Dianfu, $1 \mathrm{M}$

Li, Kang, 00

Li, Muwei, OC

Li, Zehao, 06

Lim, Marie, $1 \mathrm{~N}$

Lin, Edward P., OB

Liu, Han, 22

Liu, Tian, 1M, 1W, 29

Liu, Yingzi, IW

Livingston, Eric, 16

Lochbihler, A., 08

Long, Rodney, $1 \mathrm{E}$

Lu, Cheng, 11

Luo, Guozhen, OF

LV, Yi, 09
Lynch, Cody, OY

M. S., Vidya, 21

MacNeil, Jonathan L., OJ

Madabhushi, Anant, 11, 12

Mahajan, Vidur, 21

Mallya, Yogish, 21

Manohar, Ashish, $1 \mathrm{~K}$

Mao, Hui, 1W, 29

Marin, Angela S., $1 Y$

Marron, J.S., 16, 18

Martinez-Torteya, Antonio, OU

Matheson, Alexander M., OJ

Mayer, Michael, $1 \mathrm{~N}$

McCollough, Cynthia, 1A

McCormack, David G., OH, OJ

McCormick, Matthew, 16, 18

McDonnell, Jolie, 2A

McDonough, Joseph M., 1B

McVeigh, Elliot, $1 \mathrm{~K}$

Mertzanidou, Thomy, $1 \mathrm{G}$

Metaxas, Dimitris, 00

Michoud, Loic, OT

Miguez, Sofia, $1 \mathrm{~N}$

Mirniaharikandehei, Seyedeh-Nafiseh, 04

Mitra, Sunanda, $1 \mathrm{E}$

Mitsouras, Dimitrios, $1 \mathrm{P}$

Möddel, Martin, 1V

Moreno, Ramon A., $1 Y$

Morera, H., 20

Mori, Kensaku, OL, 1 I

Moriya, Takayasu, OL

Mostapha, Mahmoud, 26

Mowery, Y. M., $1 \mathrm{H}$

Müller, Mareike, 2B

Murphy, Ethan K., 2C

Nakamura, Shota, OL

Nakano, Y., OG

Narita, Yuji, 11

$\mathrm{Ng}$, Jia hua, $\mathrm{OQ}, \mathrm{OR}$

Niethammer, Marc, OK

Niki, N., OG

Nishankar, H., $1 \mathrm{~J}$

Nomura, Cesar H., $1 Y$

Nutter, Brian, 1E

Oakley, Amanda, $\mathrm{OQ}$

Oda, Hirohisa, OL, 11

Oda, Masahiro, OL, 11

Ohnishi, S., OG

Okamoto, T., OG

Ong-Ly, Cathy, OJ

Pai, C.-Y., 20

Paniagua, Beatriz, OT, 16, 18

Panicheva, Daryna, 15

Park, Deric M., 25

Parmar, Harshit S., $1 \mathrm{E}$

Parraga, Grace, OH, OJ

Pascal, Zille, ox

Pereira, Danilo, OV

Peterson, M. J., 20

Pettus, Jason, 2C 
Pham, Dzung L., 25

Pham, Kevin, OK

Podgorsak, A. R., 02

Polosecki, Pablo, 2A

Prakash, V., OS

Prasanna, Prateek, 11, 12

Prieto, Juan Carlos, OK, OT, 26

Prince, Jerry L., ON

Prothero, Jack, 16, 18

Pu, Jiantao, 22

Qiao, Yuting, 00

Quera-Bofarull, Arnau, 12

Quevedo Gonzalez, F. J., 17

Qureshi, Touseef Ahmad, OY

Rajabzadeh-Oghaz, Hamidreza, $1 \mathrm{~F}$

Rajapakse, Chamith S., 1N

Rajendran, Kishore, 1A

Ray, Bappaditya, OM

Reinen, Jenna, 2A

Renisch, S., OZ

Reza, Syed M. S., 25

Rish, Irina, 2A

Rittner, Letícia, OV

Rivera-Dávila, Mónica, oU

Robertsson, Vidar, ON

Rodríguez-Cantú, Félix E., OU

Rogers, Baxter P., OC

Root, Brandon K., OW

Roth, Holger R., OL, 11

Roy, Snehashis, 25

Rudin, Stephen, 02, 0A, 1J, 1P

Ruellas, Antonio Carlos, OT, 18

Rybicki, Frank J., 1P

Saito, K., OG

Sakai, H., OG

Salamon, Johannes, $1 \mathrm{~V}$

Sarkar, S., 20

Schilling, Kurt, OF

Schluchter, Andrew, $1 \mathrm{~K}$

Schmidtlein, C. Ross, 03

Schned, Alan R., 2C

Schönherr, Holger, 2B

Schulte, Gregor, 2B

Schulz, H., OZ

Schweser, Ferdinand, $1 \mathrm{~F}$

Scuffham, J., OS

Sehnert, W., OP

Setlur Nagesh, S. V., $1 \mathrm{~J}$

Shakoor, D., OP

Shankar, A., 02, OA, 1J

Shao, Muhan, ON

Sharma, Sumit, 11, 12

Shepard, Lauren M., 14, 1P

Shimao, Daisuke, 11

Shu, Hui-Kuo, IW

Siddiqui, Adnan H., 14, $1 \mathrm{~F}$

Siewerdsen, J. H., OP, 17

Sigurdsson, Sigurdur, ON

Sin, Don D., Ol

Smith, R., OS
Snyder, Kenneth V., 14

Sommer, Kelsey N., 1P

Song, Yingnan, 10

Sorci, Olivia, $1 \mathrm{~N}$

Souza, Roberto, OV

Spezi, E., OS

Srivastava, Sunil, 11, 12

Stadelmann, J. V., $0 Z$

Stepniewska, Iwona, OF

Stoyanov, Danail, $1 \mathrm{G}$

Styner, Martin, OK, OT, 26

Subramanian, S., 17

Sugino, Takaaki, 11

Sun, Changjian, 1B

Sunaguchi, Naoki, 11

Tagliati, Michele, OY

Tam, Anthony, Ol

Tamez-Peña, José G., OU

Tan, Chaowei, 00

Tan, Virak, 00

Tang, Haipeng, $1 \mathrm{M}$

Tao, Shengzhen, 1A

Thompson, Michael O., 03

Tian, Sibo, 1W

Tong, Yubing, 1B

Torigian, Drew A., 1B

Toyinbo, P. A., 20

Tuch, David, $1 G$

Turkistani, Alaa A., 10

Turner, James N., 03

Tutino, Vincent $M$., $1 F$

Udupa, Jayaram K., 1B

Ukwatta, Eranga, 08, 1C

Umetani, K., OG

Usami, Noriko, 11

van der Heide, U. A., OZ

Veasey, Benjamin, IT

Veckollari, Birgyl, 2B

Venugopal, Vasantha Kumar, 21

Villard, Pierre-Frédéric, 15

Vimort, Jean-Baptiste, 18

Vogelsang, L., OP

Wang, Cheng, $1 \mathrm{M}$

Wang, Jia Yang, 09

Wang, Lei, 22

Wang, Liya, 29

Wang, Siqi, 04

Wang, Tonghe, 1M, IW

Wang, Yuli, 06

Wang, Yunzhi, 04

Wang, Yu-Ping, $O X$

Wang, Zhiguo, $1 \mathrm{X}$

Weller, Dominik, IV

Wells, K., OS

Werner, René, 1V

Westcott, Andrew R., OH, OJ

White, James A., 1C

Wiegand, Raymond, $1 \mathrm{U}$

Wilson, David L., 10

Wilson, Michael F., 1 P 
Wismüller, Axel, OB, 1D

Wright, T., 17

Wu, Caiyun, 1B

Xiang, Liangzhong, 04

$\mathrm{XU}$, Jianfeng, 06

Yang, D., OP

Yang, Xiao, OK

Yang, Xiaofeng, 1M, 1W, 29

Yap, Moi Hoon, OQ, OR

Yatabe, Marilia, OT

Yorkston, J., 17

Yu, Hengjian, $1 \mathrm{X}$

Yu, Hong, OF

Yuasa, Tetsuya, 11

Zabihollahy, F., 08

Zarafshani, Ali, 04

Zbijewski, W., OP, 17

Zhang, Jian, 22

Zhang, Lei, 09

Zhang, Min, 09

Zheng, Bin, 04, OM

Zhou, Weihua, $1 \mathrm{M}$

Zhou, Xiangrong, $1 \mathrm{X}$

$\mathrm{Zu}$, Zhongliang, $\mathrm{OC}$ 


\section{Conference Committee}

Symposium Chairs

Ronald M. Summers, National Institutes of Health Clinical Center (United States)

Georgia D. Tourassi, Oak Ridge National Laboratory (United States)

Conference Chairs

Barjor Gimi, Cooper Medical School, Rowan University (United States) Andrzej Krol, SUNY Upstate Medical University (United States)

Conference Program Committee

Amir A. Amini, University of Louisville (United States)

Juan R. Cebral, George Mason University (United States)

Alejandro F. Frangi, University of Leeds (United Kingdom)

Xavier Intes, Rensselaer Polytechnic Institute (United States)

Ciprian N. Ionita, University at Buffalo Canon Stroke and Vascular Research Center (United States)

Vikram D. Kodibagkar, Arizona State University (United States)

Changqing Li, University of California, Merced (United States)

Armando Manduca, Mayo Clinic College of Medicine (United States)

Robert C. Molthen, GE Healthcare (United States) and Marquette University (United States) and Medical College of Wisconsin (United States)

Nicholas J. Tustison, University of Virginia (United States)

John B. Weaver, Dartmouth Hitchcock Medical Center (United States) Axel Wismüller, University of Rochester Medical Center (United States)

Baohong Yuan, The University of Texas at Arlington (United States)

\section{Session Chairs}

1 Novel Imaging Techniques and Applications I

Andrzej Krol, SUNY Upstate Medical University (United States)

Vikram D. Kodibagkar, Arizona State University (United States)

2 Keynote and Optical/Vascular I

Barjor Gimi, Cooper Medical School, Rowan University (United States)

Andrzej Krol, SUNY Upstate Medical University (United States)

3 Neurological Imaging I

Axel Wismüller, University of Rochester Medical Center (United States)

Vikram D. Kodibagkar, Arizona State University (United States) 
Andrzej Krol, SUNY Upstate Medical University (United States)

Armando Manduca, Mayo Clinic College of Medicine (United States)

5 Innovations in Image Processing I

Vikram D. Kodibagkar, Arizona State University (United States)

Nicholas J. Tustison, University of Virginia (United States)

6 Innovations in Image Processing II

Vikram D. Kodibagkar, Arizona State University (United States)

Nicholas J. Tustison, University of Virginia (United States)

$7 \quad$ Neurological Imaging II

Axel Wismüller, University of Rochester Medical Center (United States)

Nicholas J. Tustison, University of Virginia (United States)

8 Optical/Vascular II

Ciprian N. Ionita, University at Buffalo Canon Stroke and Vascular Research Center (United States)

9 Bone

Andrzej Krol, SUNY Upstate Medical University (United States)

Baohong Yuan, The University of Texas at Arlington (United States)

$10 \mathrm{MRI}$ and $\mathrm{fMRI}$

Armando Manduca, Mayo Clinic (United States)

Andrzej Krol, SUNY Upstate Medical University (United States)

11 Novel Imaging Techniques and Applications II

Baohong Yuan, The University of Texas at Arlington (United States)

Ciprian N. Ionita, University at Buffalo Canon Stroke and Vascular Research Center (United States) 


\section{Medical Imaging Award Recipients}

\section{Robert F. Wagner Best Student Paper Award}

Robert F. Wagner was an active scientist in the SPIE Medical Imaging meeting, starting with the first meeting in 1972 and continuing throughout his career. He ensured that the BRH, and subsequently the $\mathrm{CDRH}$, was a sponsor for the early and subsequent Medical Imaging meetings, helping to launch and ensure the historical success of the meeting. The Robert F. Wagner All-Conference Best Student Paper Award (established 2014) is acknowledgment of his many important contributions to the Medical Imaging meeting and his many important advances to the field of medical imaging.

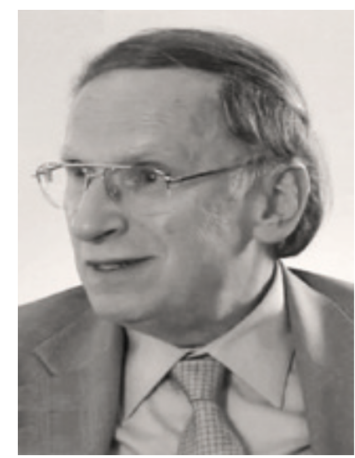

This award is co-sponsored by:

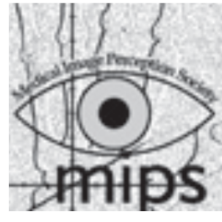

The Medical Image Perception Society

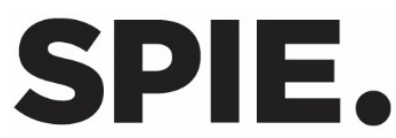

\section{Recipients:}

First Place: Volume-of-interest imaging using multiple aperture devices (10984-74) Wenying Wang, Grace J. Gang, Jeffrey H. Siewerdsen, Joseph W. Stayman, Johns Hopkins University (United States)

Second Place: Surgical aid visualization system for glioblastoma fumor identification based on deep learning and in-vivo hyperspectral images of human patients (10951-35)

Himar Fabelo, The University of Texas at Dallas (USA) and Universidad of Las Palmas de Gran Canaria (Spain); Martin Halicek, The University of Texas at Dallas (United States) and Georgia Institute of Technology \& Emory University School of Medicine (United States); Samuel Ortega, Universidad de Las Palmas de Gran Canaria (Spain); Adam Szolna, Jesus Morera, Hospital Universidad de Gran Canaria Doctor Negrin (Spain); Roberto Sarmiento, Universidad of Las Palmas de Gran Canaria (Spain); Gustavo M. Callicó, Universidad de Las Palmas de Gran Canaria (Spain); Baowei Fei, The University of Texas at Dallas (United States) and The University of Texas Southwestern Medical Center (United States) 
Proc. of SPIE Vol. 10953 1095301-16

Downloaded From: https://www.spiedigitallibrary.org/conference-proceedings-of-spie on 26 Apr 2023 Terms of Use: https://www.spiedigitallibrary.org/terms-of-use 


\section{Introduction}

The 2019 SPIE "Biomedical Applications in Molecular, Structural and Functional Imaging" Conference was held on 19 - 21 February, 2019 at Town and Country Resort, San Diego, California, United States. We maintained the high participation we saw last year, both in the number of abstracts submitted and in the conference attendees over previous years. Conference Chairs Barjor Gimi and Andrzej Krol welcomed Professor Christopher Filippi, North Shore-Long Island Jewish Medical System and Columbia University (USA), who delivered an outstanding, insightful keynote address entitled "The dawning of Al in radiology: a brave new world" that provided a brief review of machine learning and deep learning techniques in artificial intelligence (AI), as applied in diagnostic radiology, with the focus on the translation of $\mathrm{Al}$ into diagnostic radiology from clinical workflow to its implementation in routine clinical practice to informed diagnosis, treatment management, and prognostication. Specific, ongoing work in the automated detection of hemorrhage on non-contrast head CT, prediction of genetic variability of brain tumors, detection of breast cancer and risk factors for breast cancer, and detection of knee ligament injury was profiled among other clinical applications. Both machine learning and deep learning techniques are transforming how radiologists make intelligent decisions from the quantitative diagnostic images that will require that radiologists become data scientists and data managers in the future.

The diverse sessions included Keynote, Bone Imaging, Cardiovascular Imaging, Innovations in Image Processing, MRI and fMRI, Neurological Imaging, Novel Imaging Techniques and Applications, Optical, Pulmonary and Vascular Imaging.

We are grateful to the chairs of sessions: Ciprian Ionita, Vikram Kodibagkar, Armando Manduca, Nicholas Tustison, Axel Wismuller, and Baohong Yuan.

The poster session comprised vibrant discussions. A panel of judges selected "Machine-learning based classification of glioblastoma using dynamic susceptibility enhanced MR image" by Jiwoong Jason Jeong et al. (Emory University, USA) for the Cum Laude award.

The poster "Brain MRI classification based on machine learning framework with auto-context mode" by Yang Lei et al. (Emory University, USA), was selected as an Honorable Mention.

Gabrielle M. Colvert (University of California San Diego) was selected as $1^{\text {st }}$ finalist for the paper entitled "Novel measurement of LV twist using 4DCT: quantifying accuracy as a function of image noise" for Robert F. Wagner All-Conference Best Student Paper Awards. 
Joseph Bullock (Durham University) was selected as $2^{\text {nd }}$ finalist for the paper entitled "XNet: a convolutional neural network (CNN) implementation for medical x-ray image segmentation suitable for small datasets" for Robert F. Wagner AllConference Best Student Paper Awards.

Andrzej Krol Barjor Gimi 